Supporting Information for:

\title{
Synthesis of Emissive Heteroacene Derivatives via Nucleophilic Aromatic Substitution
}

Lana K. Hiscock, ${ }^{\dagger}$ Chengzhang Yao,${ }^{\ddagger}$ W. G. Skene, ${ }^{\ddagger}$ Louise N. Dawe, ${ }^{\dagger}$ and Kenneth E. Maly* ${ }^{\dagger}$

$\dagger$ Department of Chemistry and Biochemistry, Wilfrid Laurier University, 75 University Ave. W., Waterloo, ON, N2L 3C5, Canada

†Département de Chimie, Université de Montréal, CP 6128, Centre-ville, Montreal, QC, H3C 3J7, Canada

*To whom correspondence should be addressed. E-mail: kmaly@wlu.ca

\section{Contents:}

${ }^{1} \mathrm{H}$ and ${ }^{13} \mathrm{C}$ NMR Spectra 


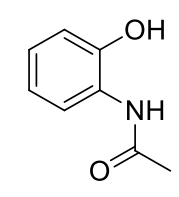

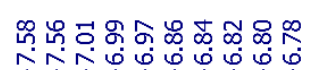

11b

1H NMR, $400 \mathrm{MHz}$

MeOD

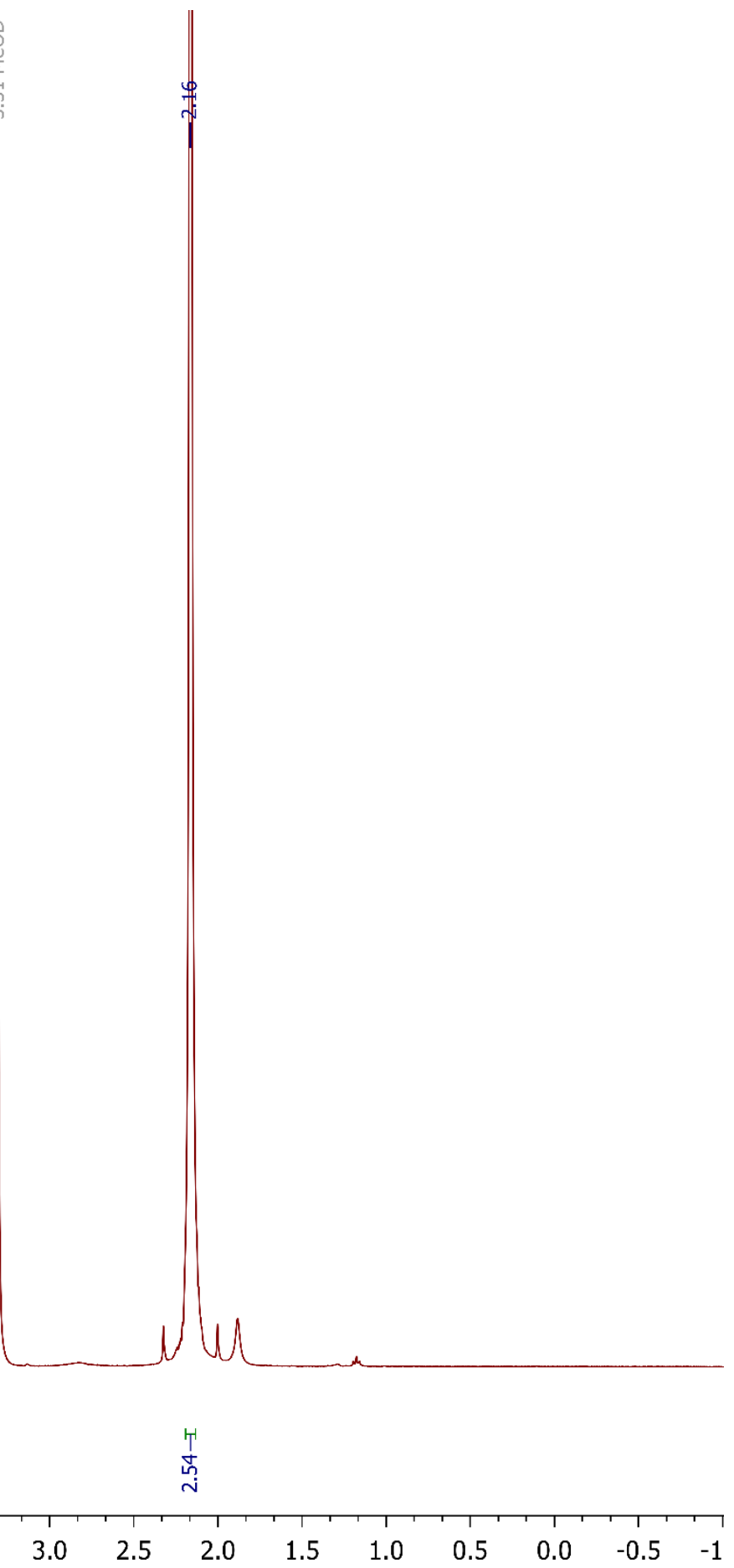


${ }^{13} \mathrm{CNMR}\left(100 \mathrm{MHz}, \mathrm{cd}_{3} \mathrm{Od}\right) \delta 172.20,149.72,126.80,123.96,120.60,117.27,23.43$

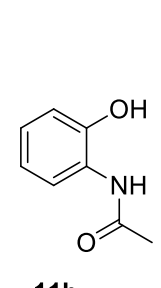

11b

13C NMR, $100 \mathrm{MHz}$

$\mathrm{MeOD}$

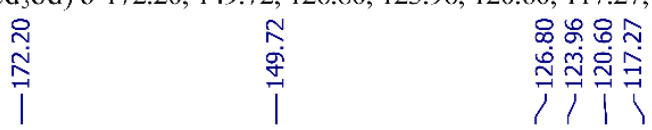

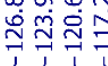

111 

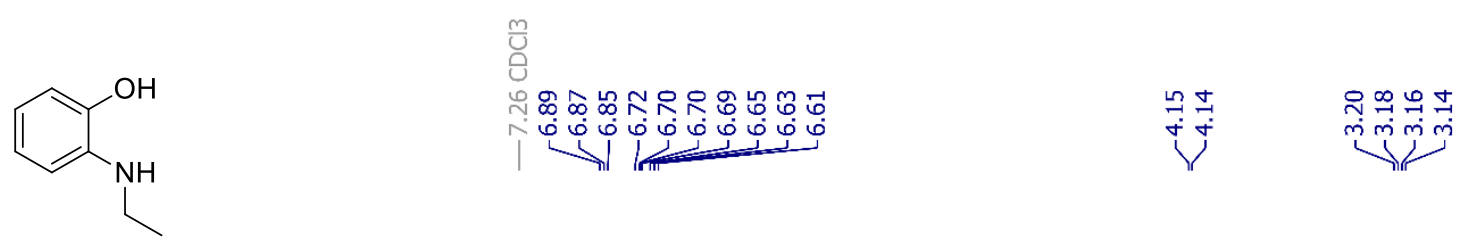

12b

$1 \mathrm{H}$ NMR, $400 \mathrm{MHz}$

$\mathrm{CDCl}_{3}$

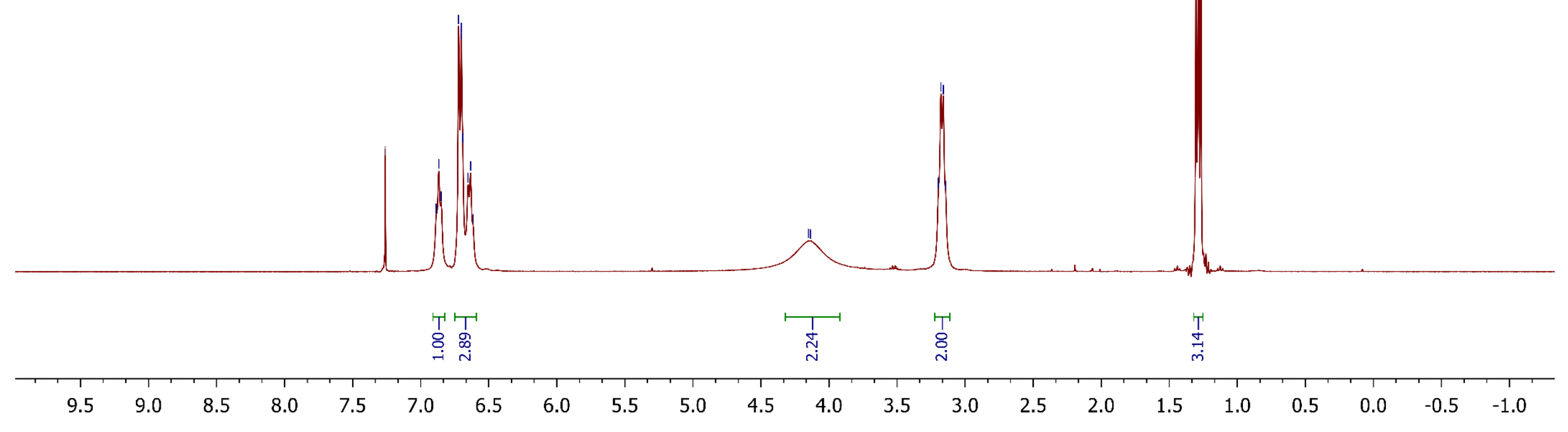


${ }^{13} \mathrm{C} \mathrm{NMR}\left(100 \mathrm{MHz}, \mathrm{cdcl}_{3}\right) \delta 143.92,137.26,121.76,117.98,114.45,112.87,39.13,15.05$.

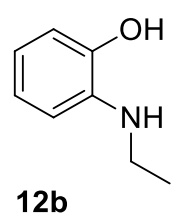

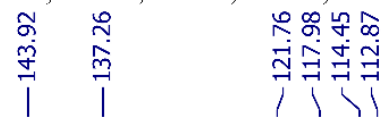

$12 b$

13C NMR, $100 \mathrm{MHz}$

$\mathrm{CDCl}_{3}$

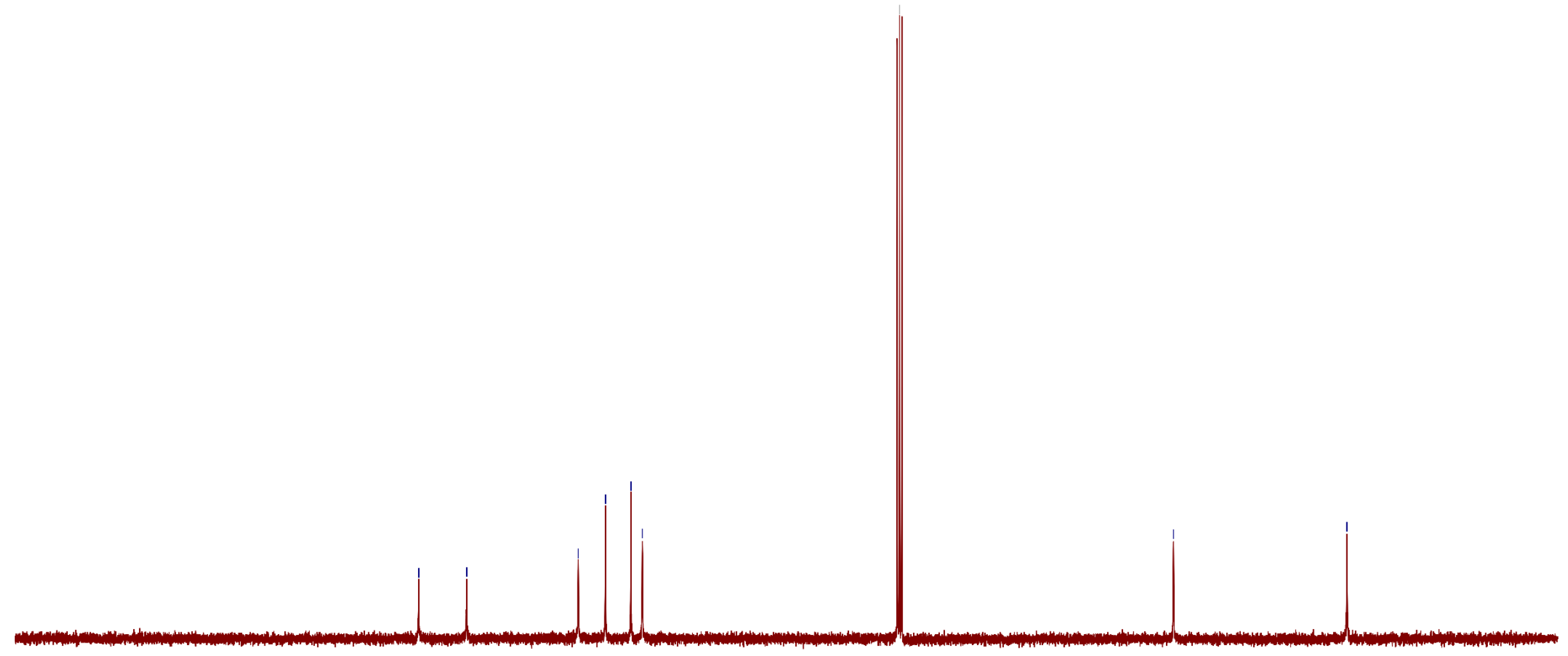




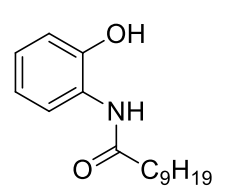

$11 \mathrm{c}$

$1 \mathrm{H} \mathrm{NMR}, 400 \mathrm{MHz}$

$\mathrm{CDCl} 3$

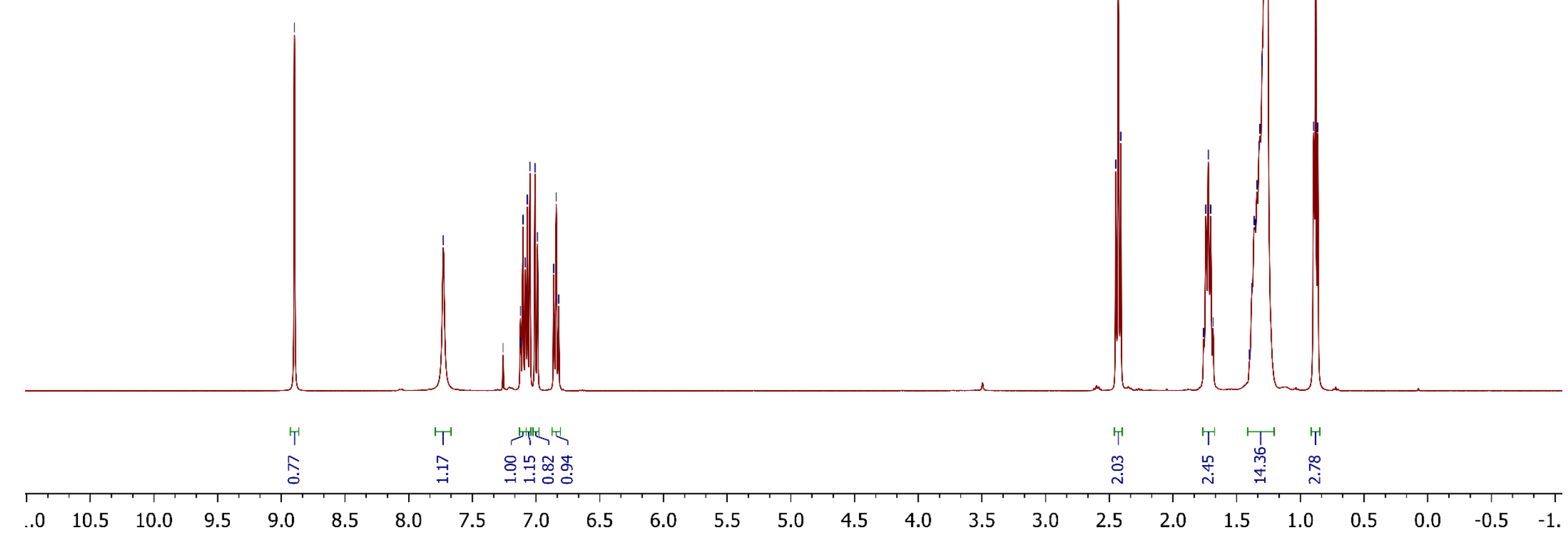


$11 \mathrm{c}$

13C NMR, $100 \mathrm{MHz}$

$\mathrm{CDCl}_{3}$

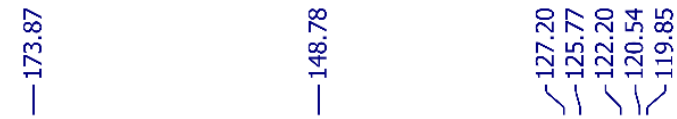

${ }^{13} \mathrm{C} \mathrm{NMR}\left(100 \mathrm{MHz}, \mathrm{cdcl}_{3}\right) \delta 173.87,148.78,127.20,125.77,122.20,120.54,119.85,37.17,31.98,29.54,29.44$, 29.39, 29.29, 25.92, 22.80, 14.24 .

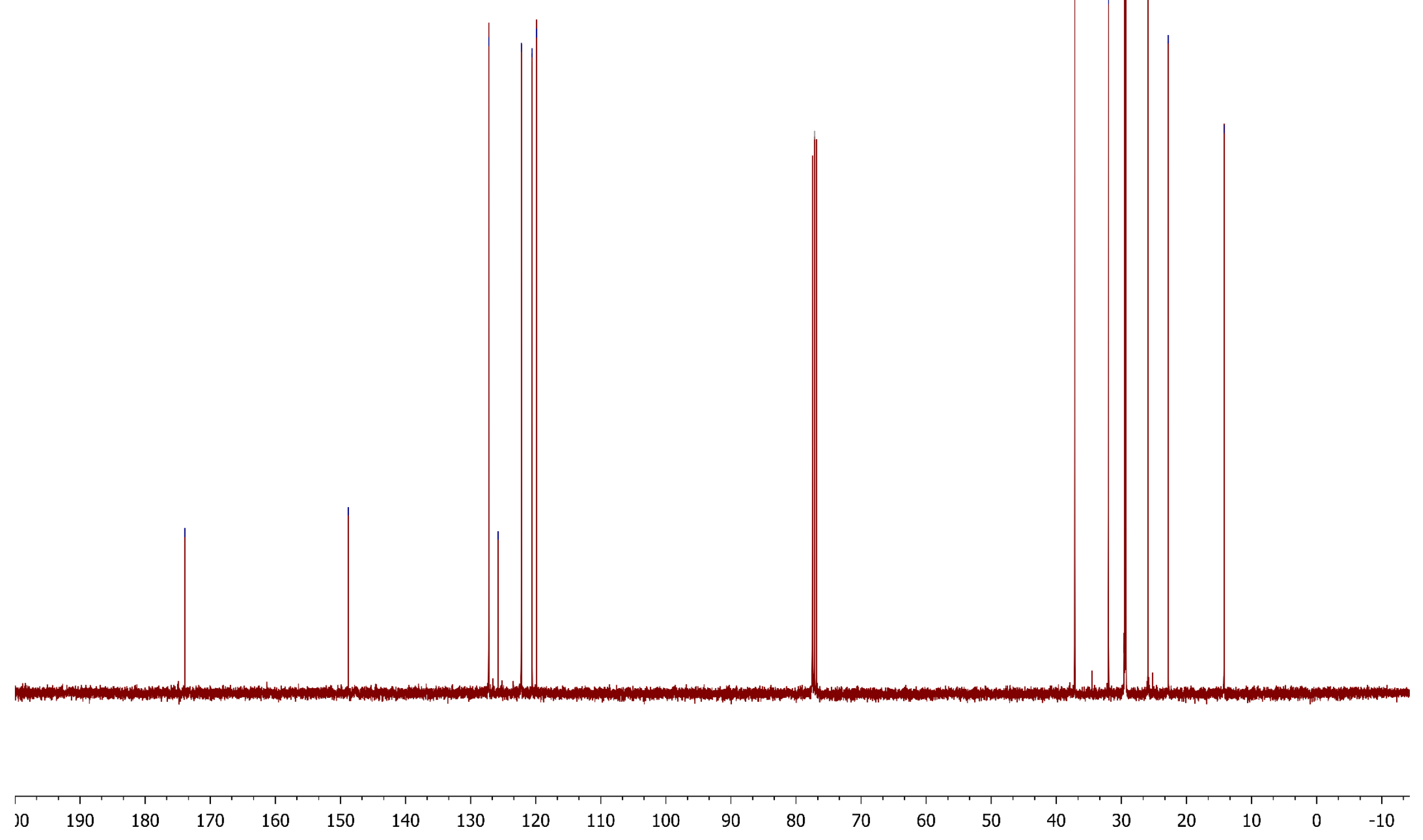




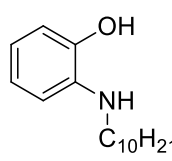

$12 \mathrm{c}$

$1 \mathrm{H} \mathrm{NMR}, 400 \mathrm{MHz}$

$\mathrm{CDCl}_{3}$
$\stackrel{m}{\mathrm{O}}$

L्ञ

ن

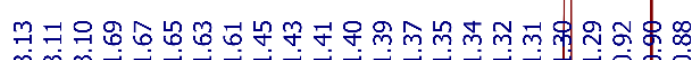
लिm
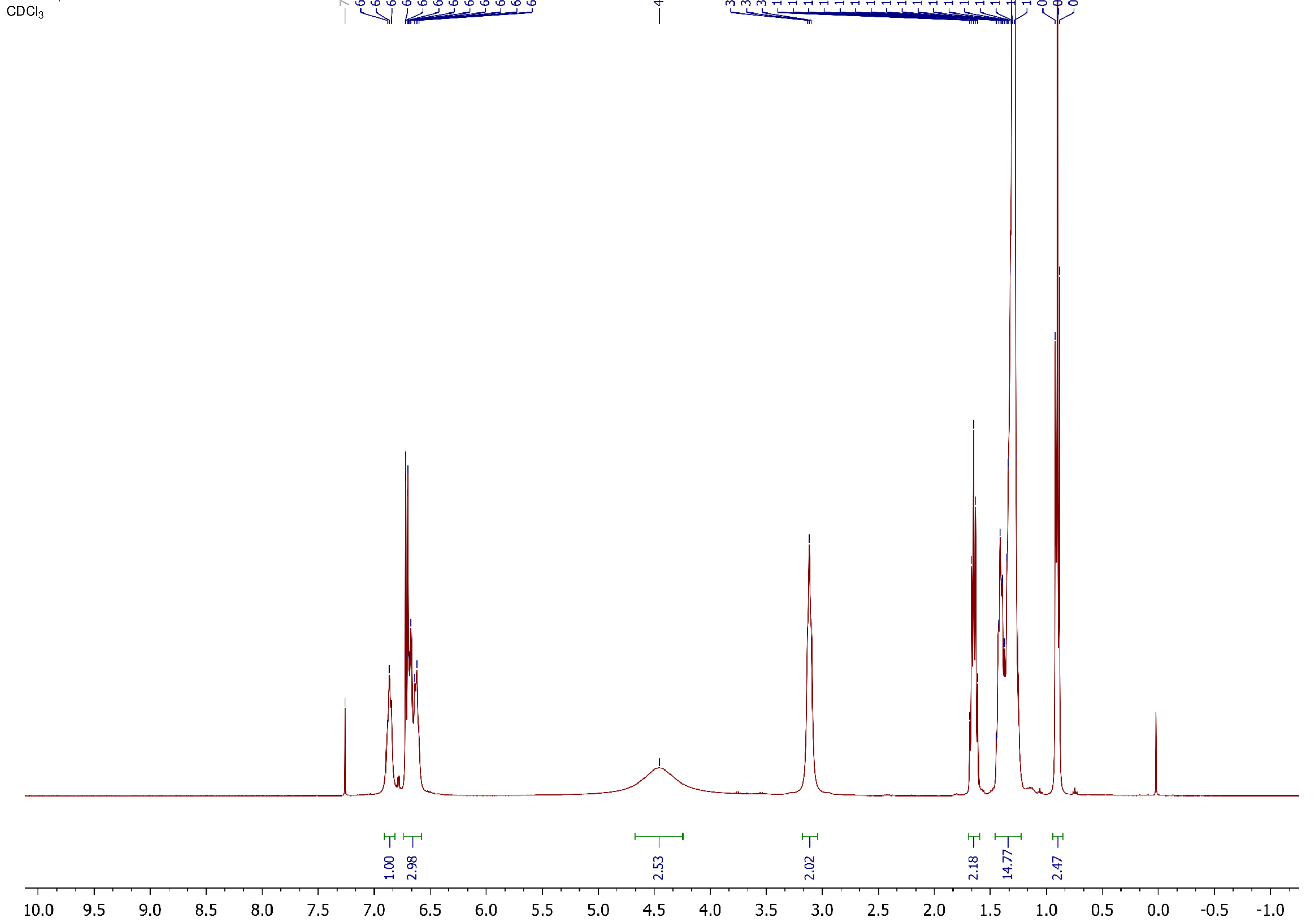
$\overbrace{\substack{\mathrm{N} \\ \mathrm{C}_{10} \mathrm{H}_{21}}}^{\mathrm{OH}}$

$12 \mathrm{c}$

13C NMR, $100 \mathrm{MHz}$

$\mathrm{CDCl}_{3}$ $m$
0
0
0

$i$

${ }^{13} \mathrm{C} \mathrm{NMR}\left(100 \mathrm{MHz}, \mathrm{cdcl}_{3}\right) \delta 143.87,137.42,121.72,117.81,114.50,112.74,44.68,32.04,29.75,29.72,29.70,29.69,29.66,29.61$,

${ }^{13} \mathrm{C} \mathrm{NMR}(100 \mathrm{MHz}, \mathrm{cdcl})$
$29.47,27.34,22.82,14.26$.

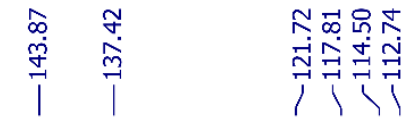

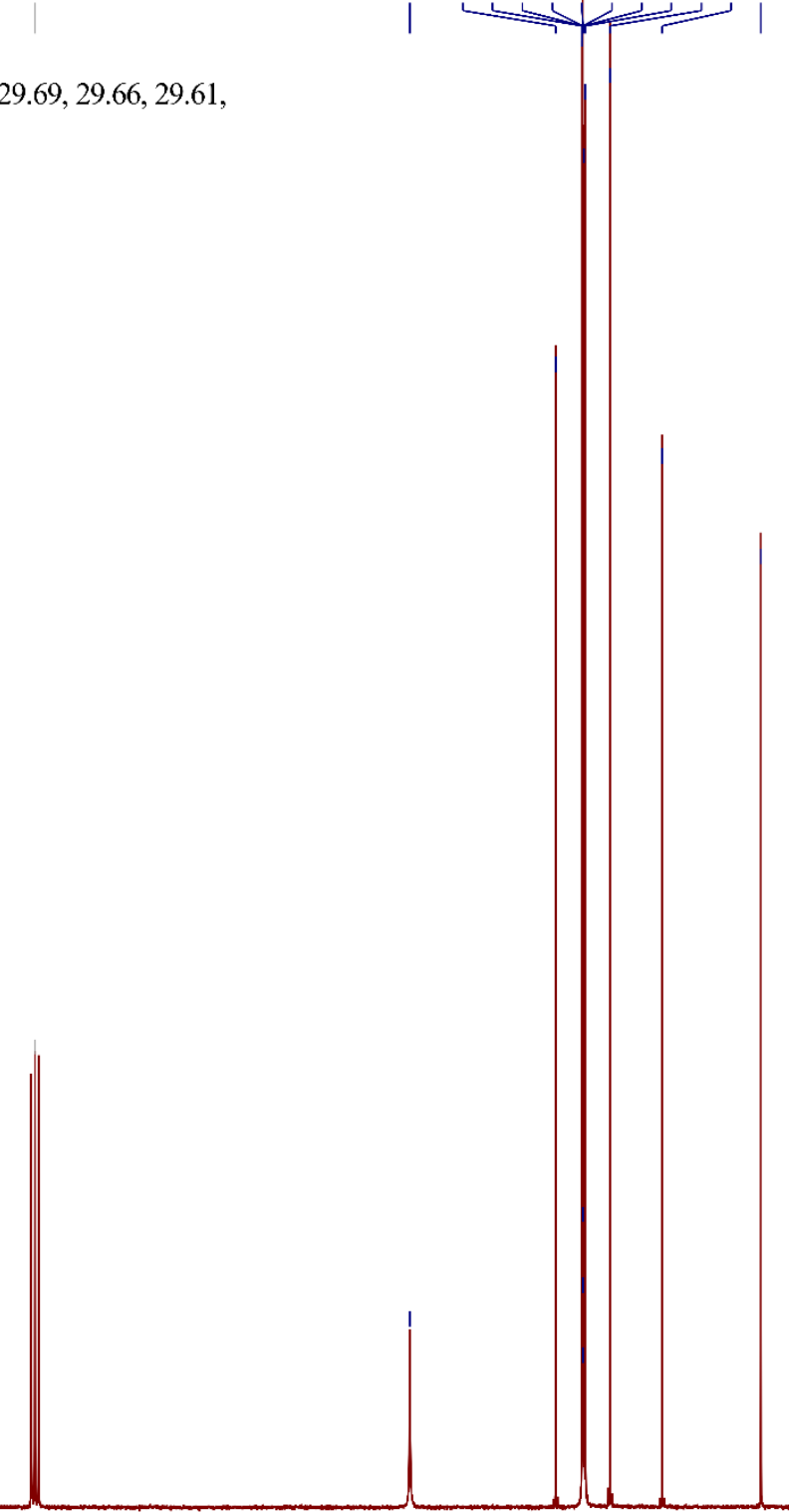

。

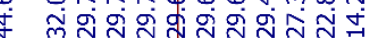

12

\begin{tabular}{|c|c|c|c|c|c|c|c|c|c|c|c|c|c|c|c|c|c|c|c|}
\hline 1 & 1 & 1 & 1 & 1 & 1 & 1 & 1 & 1 & & 1 & 1 & 1 & 1 & 1 & 1 & 1 & 1 & 1 & 1 \\
\hline 190 & 180 & 170 & 160 & 150 & 140 & 130 & 120 & 110 & 100 & 90 & 80 & 70 & 60 & 50 & 40 & 30 & 20 & 10 & -10 \\
\hline
\end{tabular}




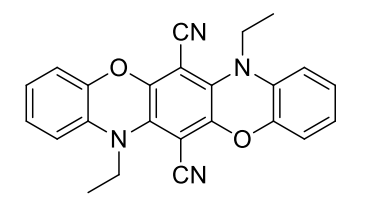

4b

1H NMR, $400 \mathrm{MHz}$
$\mathrm{CDCl} 3$

兽

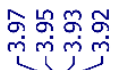

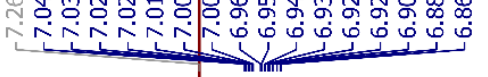

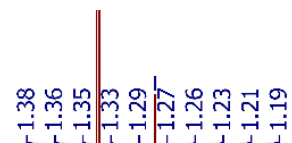

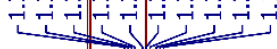

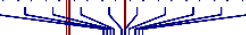

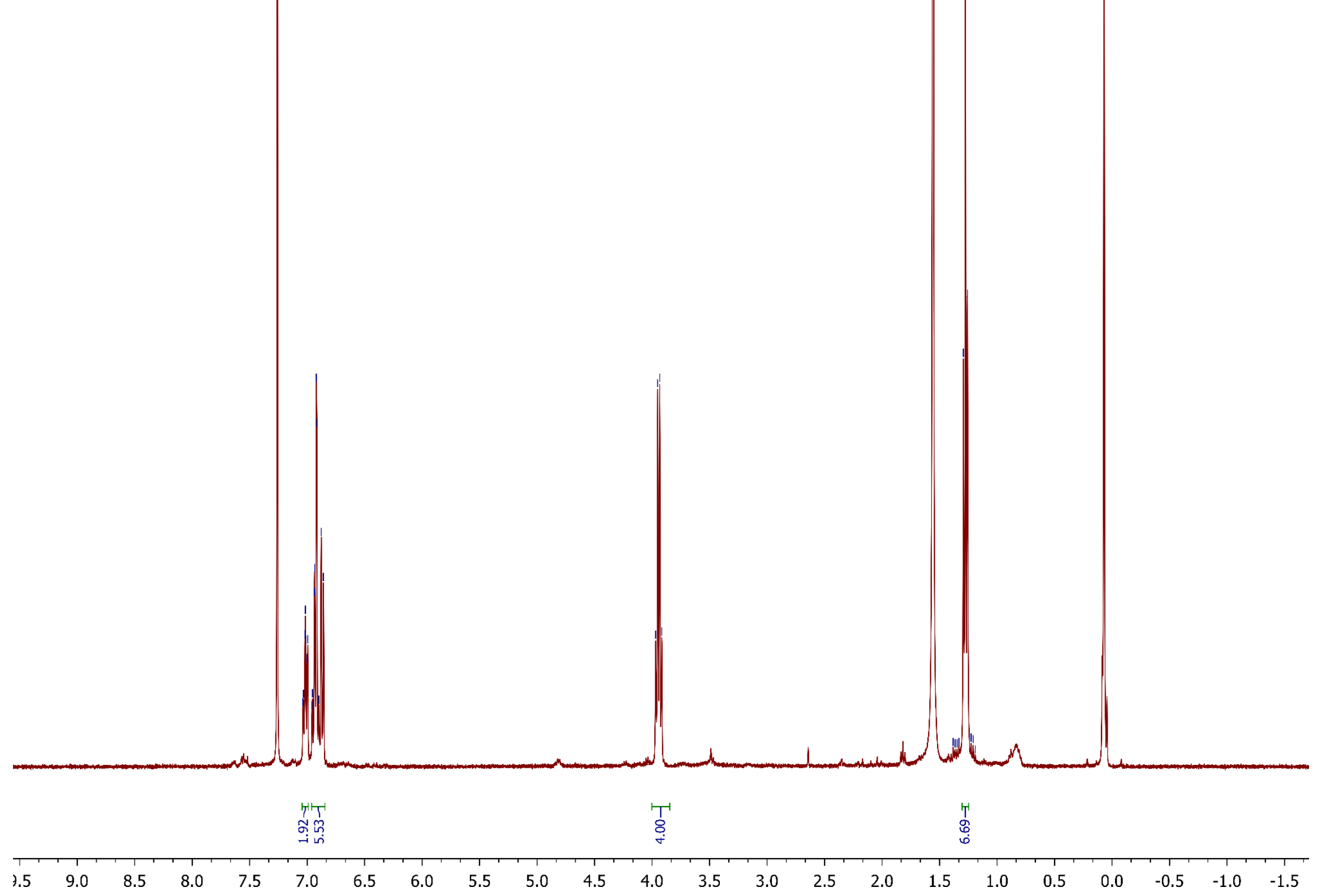





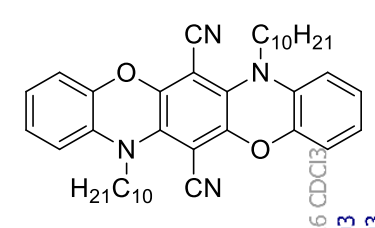

$4 c$

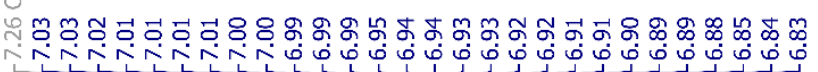

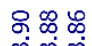

1H NMR, $400 \mathrm{MHz}$

$\mathrm{CDCl} 3$

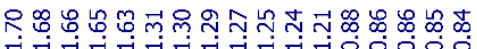
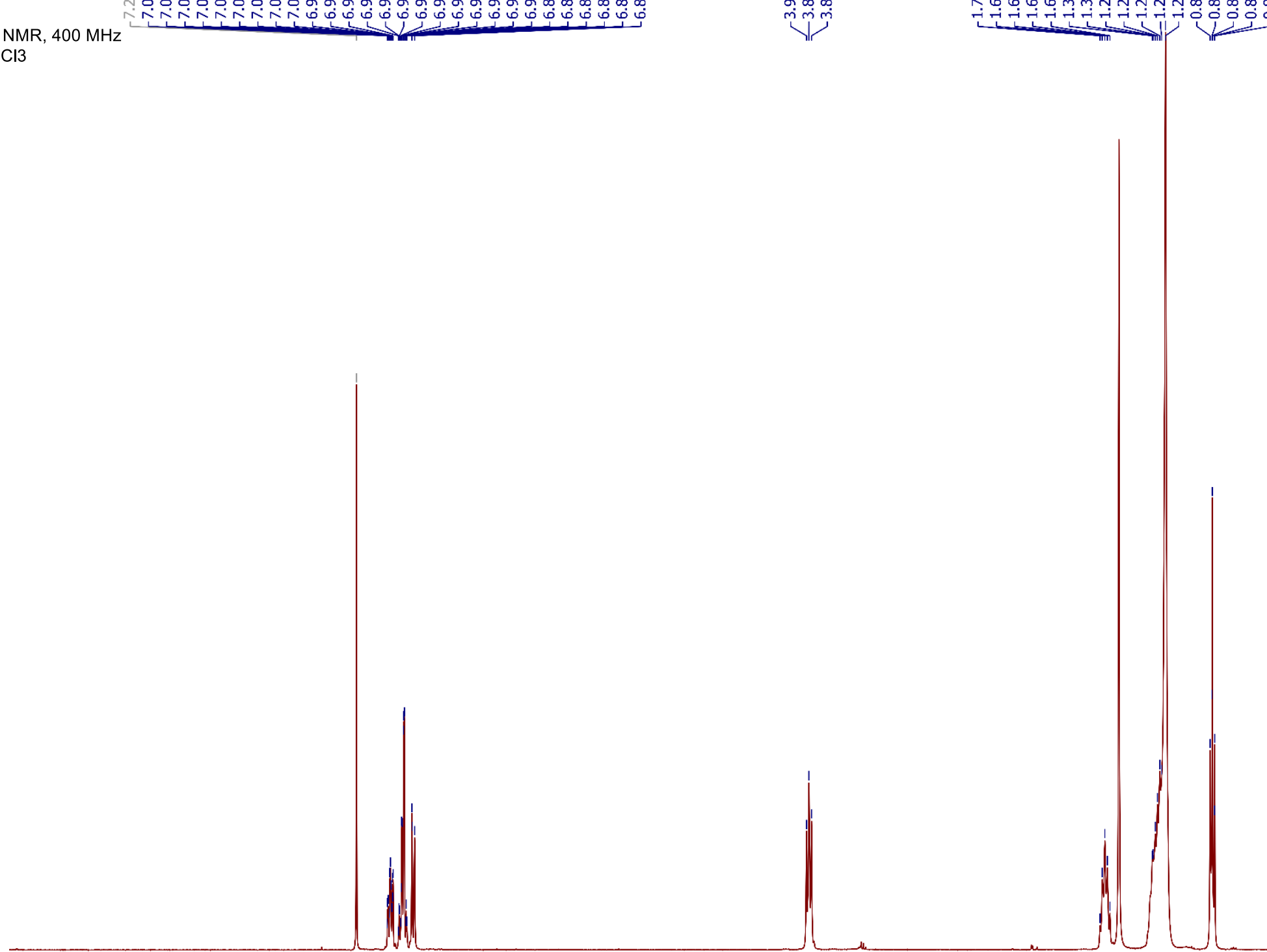

tritth

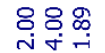

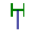

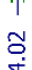

$\begin{array}{lllll}9.5 & 9.0 & 8.5 & 8.0 & 7.5\end{array}$

6.5

6.0

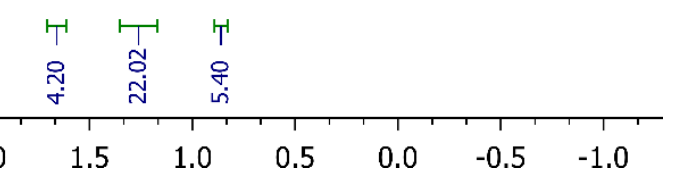


CN $\quad \mathrm{C}_{10} \mathrm{H}_{21}$

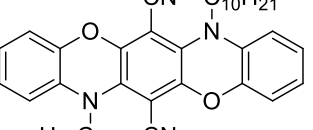

$\mathrm{H}_{21} \mathrm{C}_{10} \mathrm{CN}$

$4 \mathrm{c}$

$13 \mathrm{C} \mathrm{NMR}, 100 \mathrm{MHz}$

$\mathrm{CDCl} 3$

${ }^{13} \mathrm{CNMR}\left(100 \mathrm{MHz}, \mathrm{cdcl}_{3}\right) \delta 147.60,147.21,132.97,131.64,125.03,123.95,118.46,116.25$ $112.73,94.54,52.98,31.68,29.29,29.27,29.08,29.02,27.26,26.12,22.49,13.93$.

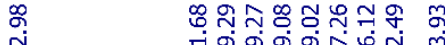

!

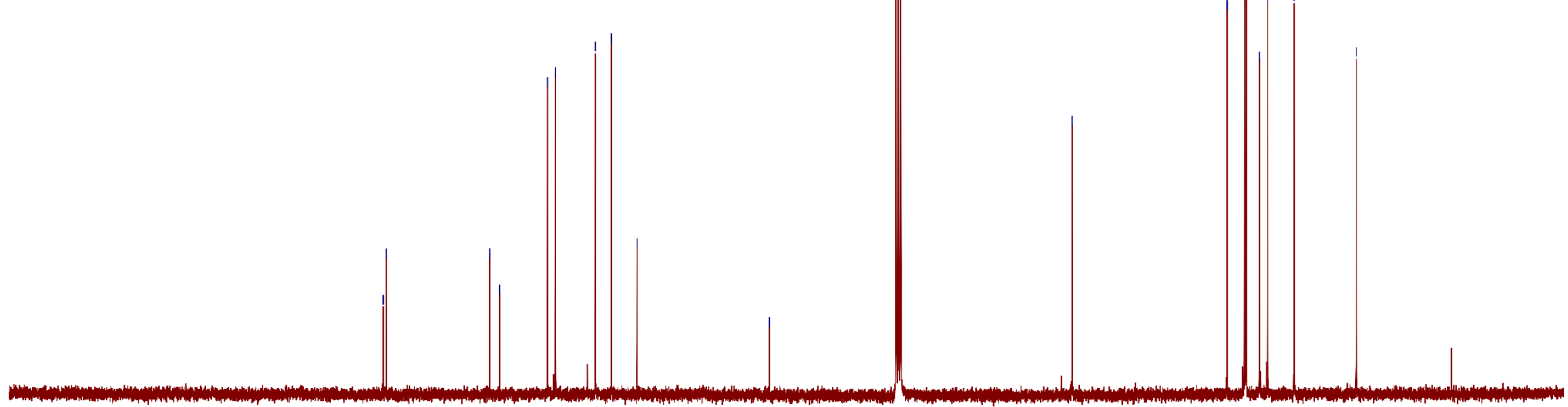

\begin{tabular}{lllllllllllllllllllllllllll}
\hline 190 & 180 & 170 & 160 & 150 & 140 & 130 & 120 & 110 & 100 & 90 & 80 & 70 & 60 & 50 & 40 & 30 & 20 & 10 & 0 & -10
\end{tabular}




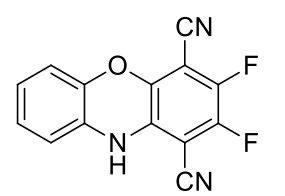

10

$13 \mathrm{C}$ NMR, $100 \mathrm{MHz}$

Acetone-d6

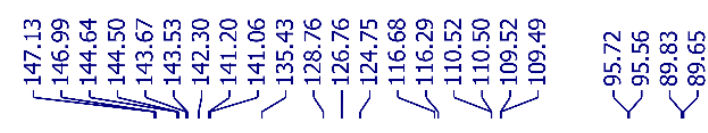

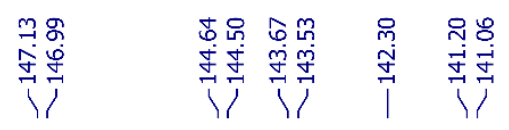

$$
\left.\begin{array}{c|c}
A(d d) \\
145.81 \\
J(250.11,13.64)
\end{array}\right] \begin{gathered}
B(d d) \\
142.37 \\
J(248.10,14.70) \\
\hline
\end{gathered}
$$

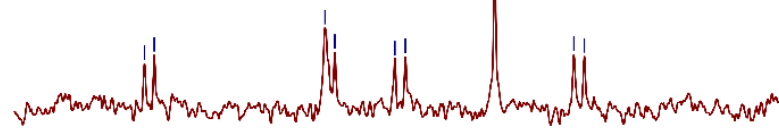

$\begin{array}{llllllllll}148 & 147 & 146 & 145 & 144 & 143 & 142 & 141 & 140 & 139\end{array}$

$\begin{array}{lllllllllllllllllllllllllll}230 & 220 & 210 & 200 & 190 & 180 & 170 & 160 & 150 & 140 & 130 & 120 & 110 & 100 & 90 & 80 & 70 & 60 & 50 & 40 & 30 & 20 & 10 & 0 & -10\end{array}$




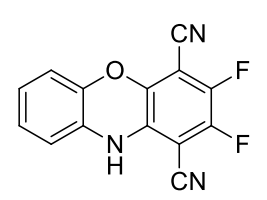

10

$19 \mathrm{~F}$ NMR, $376 \mathrm{MHz}$

Acetone-d6

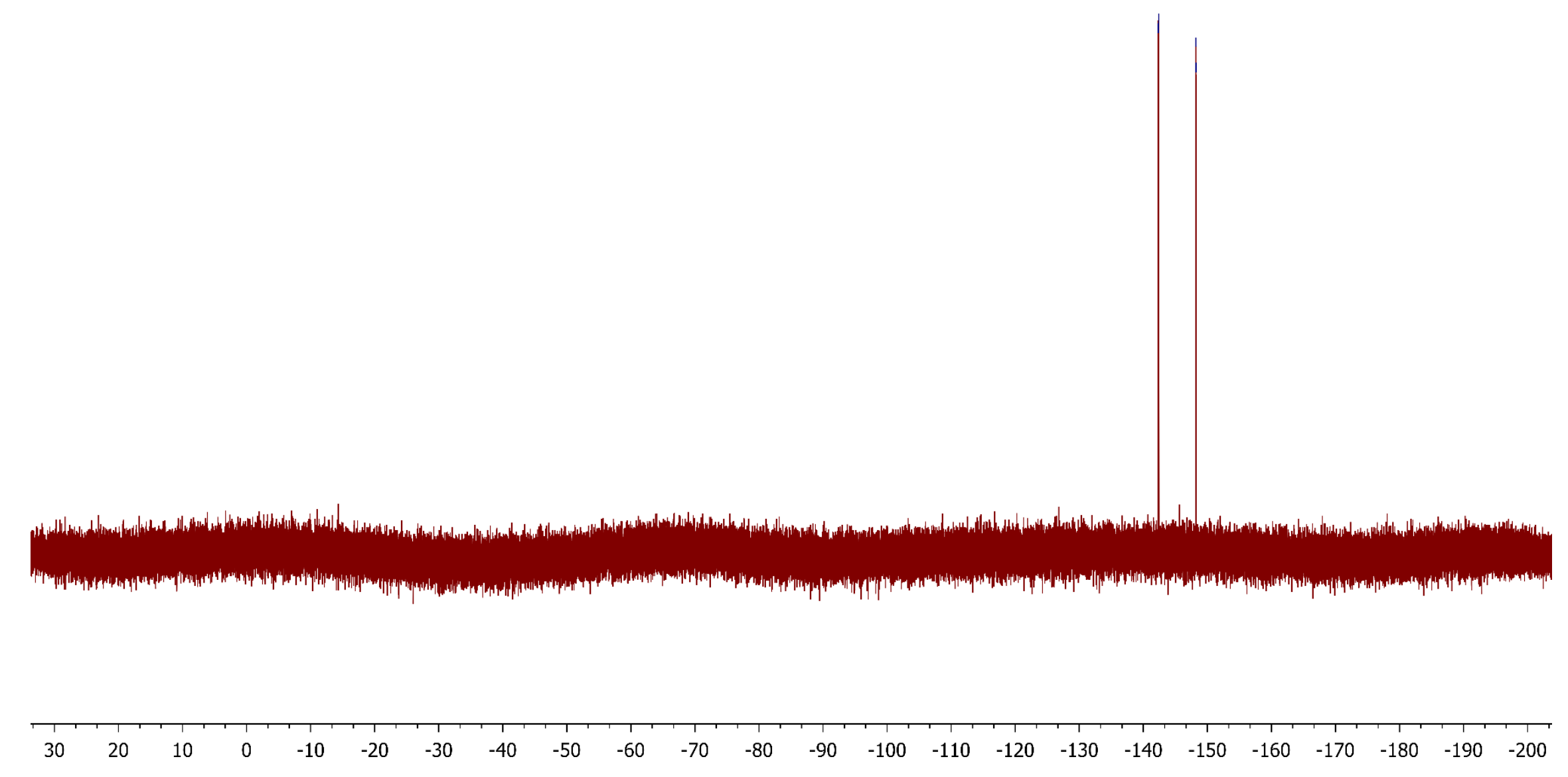




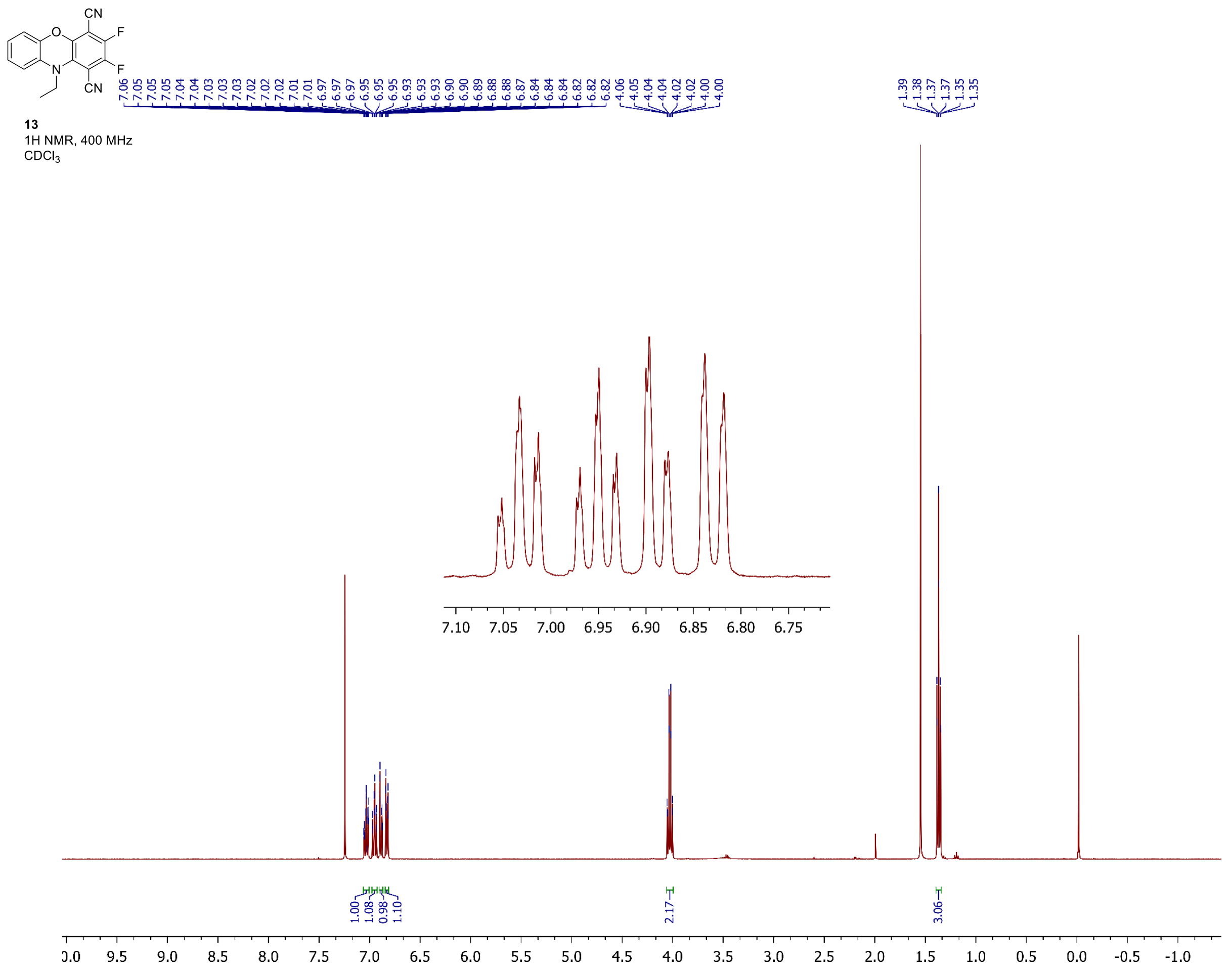




$$
\text { Tlli }
$$



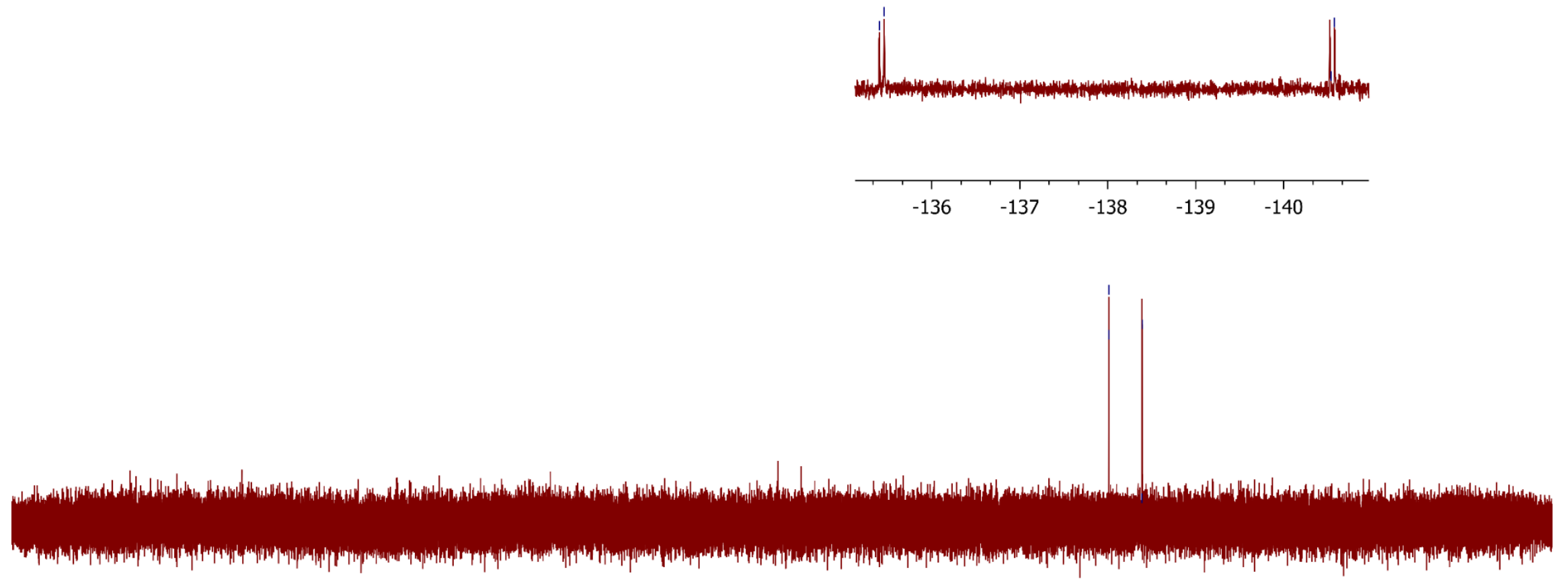
<smiles>N#Cc1c(F)c(F)c(C#N)c2c1Oc1ccccc1O2</smiles>

8

$1 \mathrm{H} \mathrm{NMR}, 300 \mathrm{MHz}$ $\mathrm{CDCl}_{3}$
嘀

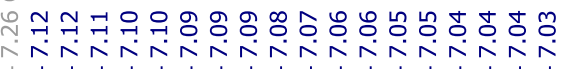

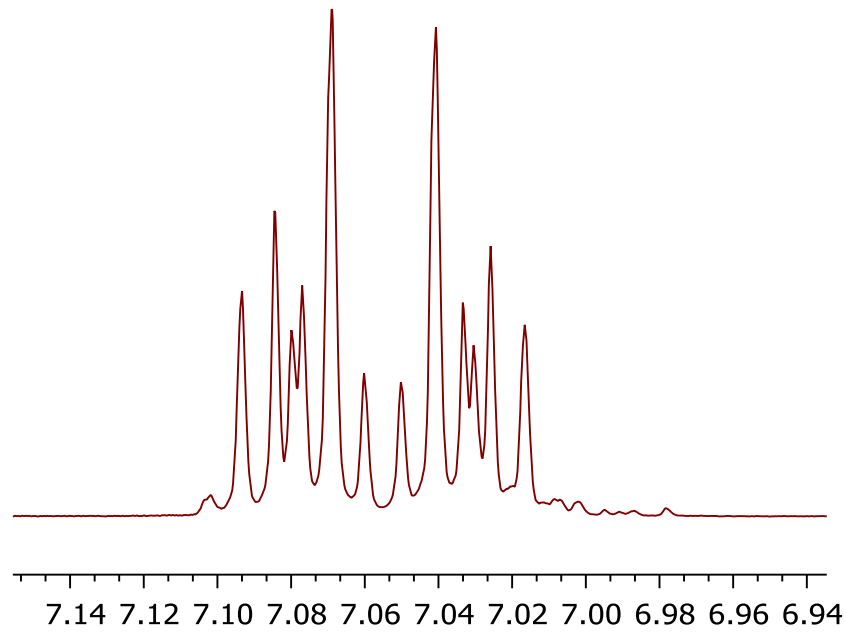

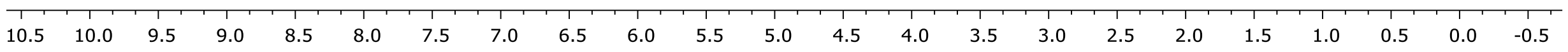




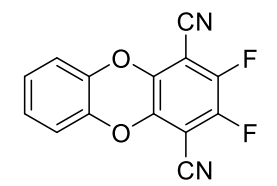

8

13C NMR, $75 \mathrm{MHz}$

$\mathrm{CDCl}_{3}$

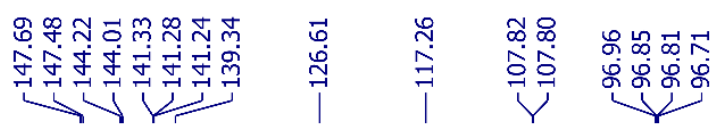

${ }^{13} \mathrm{C} \mathrm{NMR}\left(75 \mathrm{MHz}, \mathrm{cdcl}_{3}\right) \delta 147.69,147.48,144.22,144.01,141.33,141.28,141.24,139.34$, $126.61,117.26,107.82,107.80,96.96,96.85,96.81,96.71$.

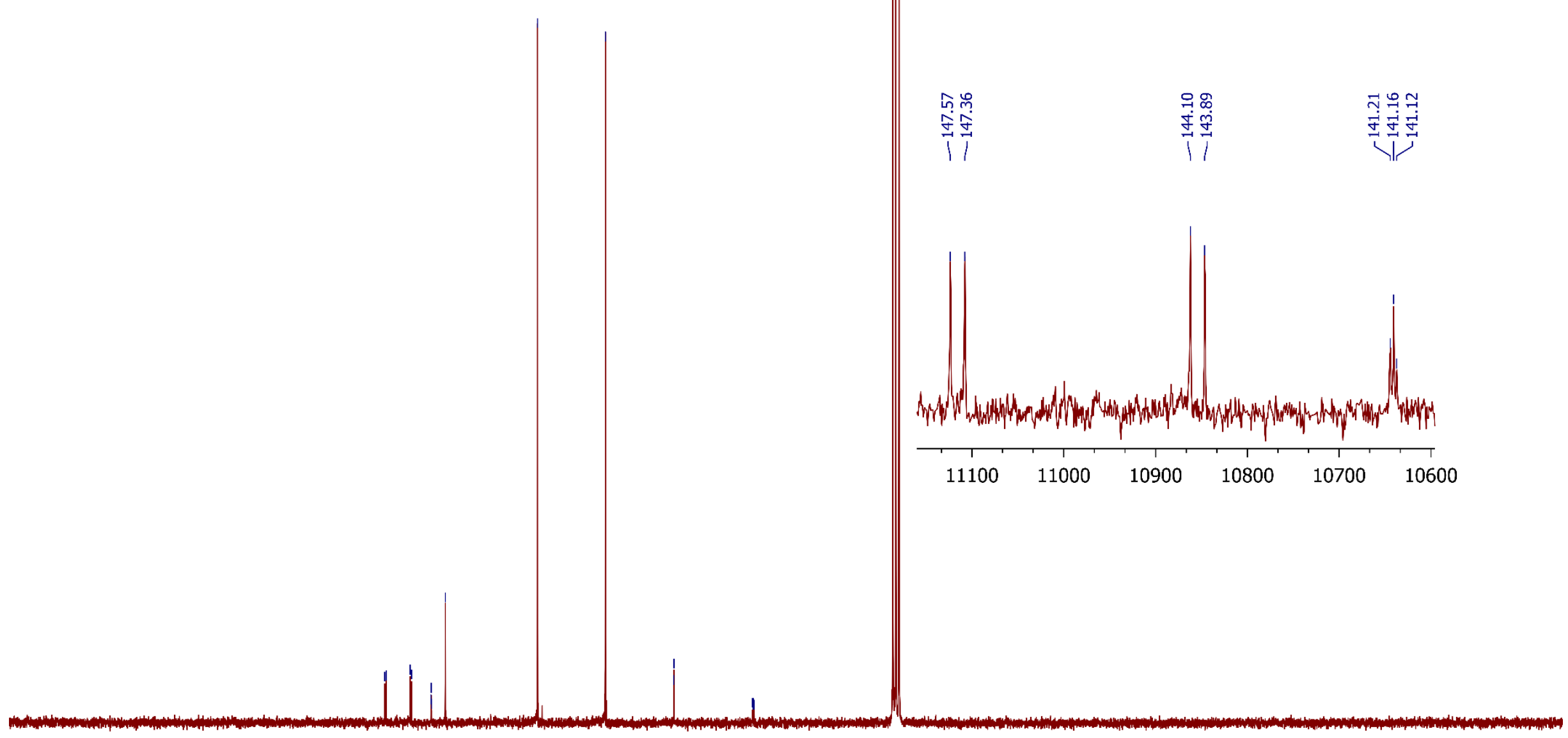


${ }^{19} \mathrm{~F} \mathrm{NMR}\left(376 \mathrm{MHz}, \mathrm{cdcl}_{3}\right) \delta-134.75$.<smiles>N#Cc1c(F)c(F)c(C#N)c2c1Oc1ccccc1O2</smiles>

8

19F NMR, $376 \mathrm{MHz}$

$\mathrm{CDCl} 3$

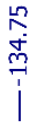

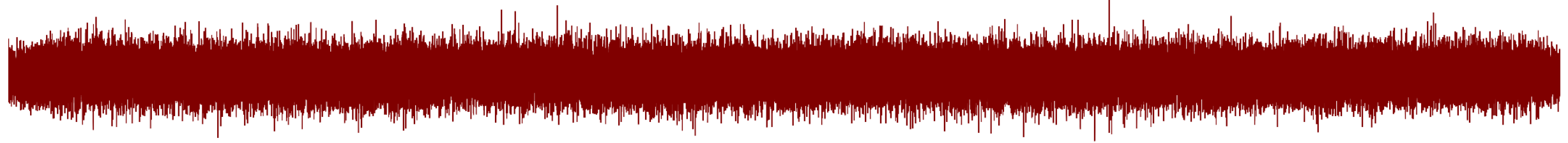

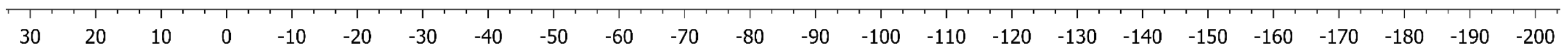



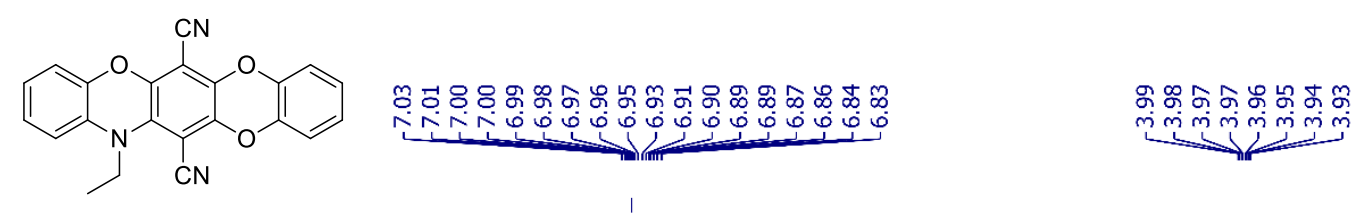

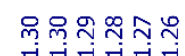

5 H NMR, $400 \mathrm{MHz}$

$\mathrm{CDCl}_{3}$

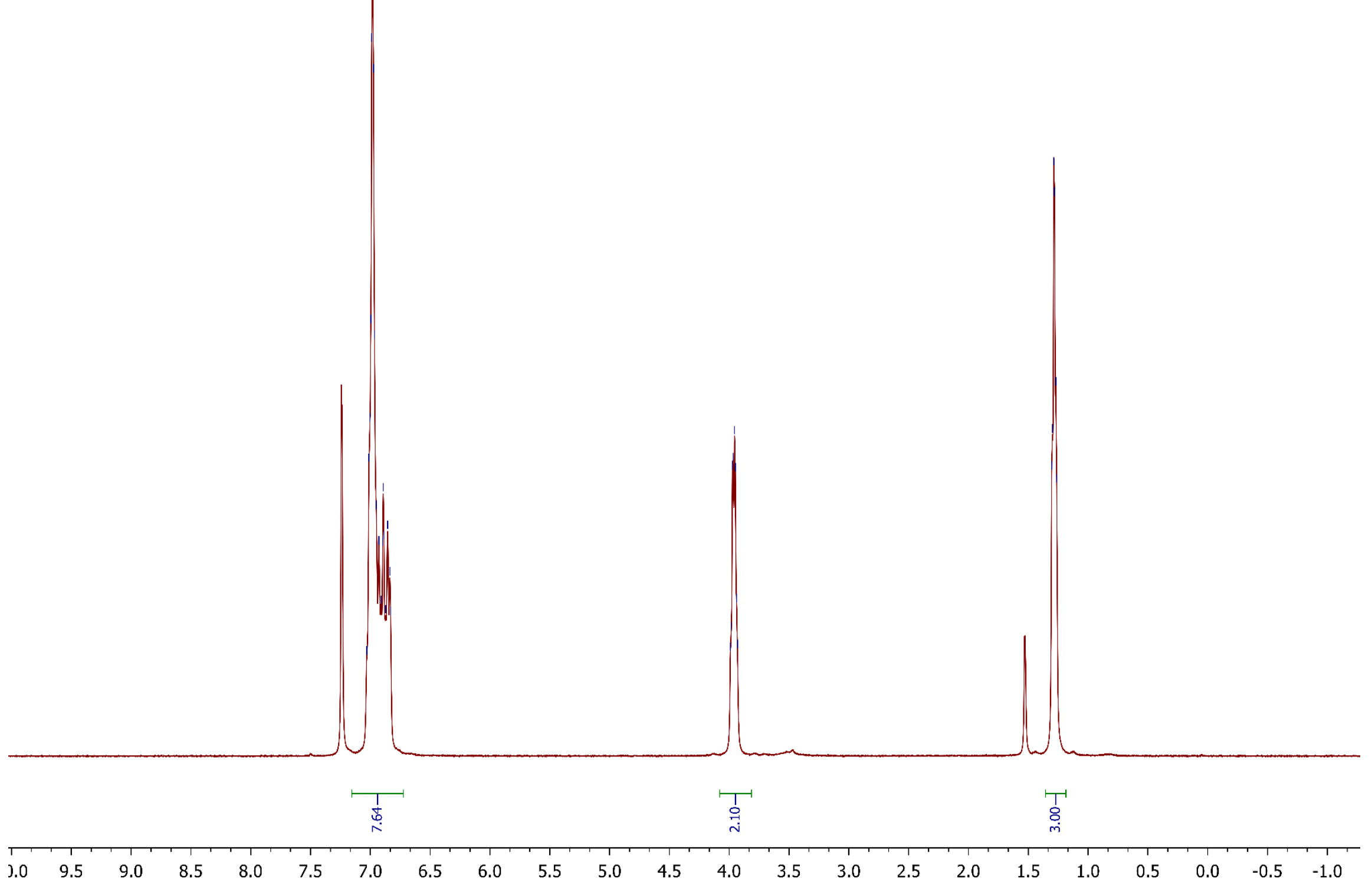




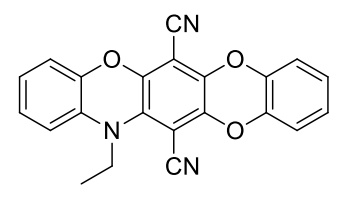

5

13C NMR, $100 \mathrm{MHz}$

${ }^{13} \mathrm{CNMR}\left(100 \mathrm{MHz}_{\mathrm{cdcl}}\right) \delta 147.15,146.91,140.32,140.20,139.84,137.30,133.79,132.23$ $125.99,125.66,125.63,124.72,118.61,117.08,117.00,116.54,112.35,110.12,94.40,94.26$, $48.40,13.37$.

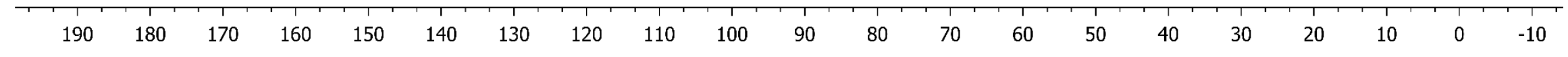




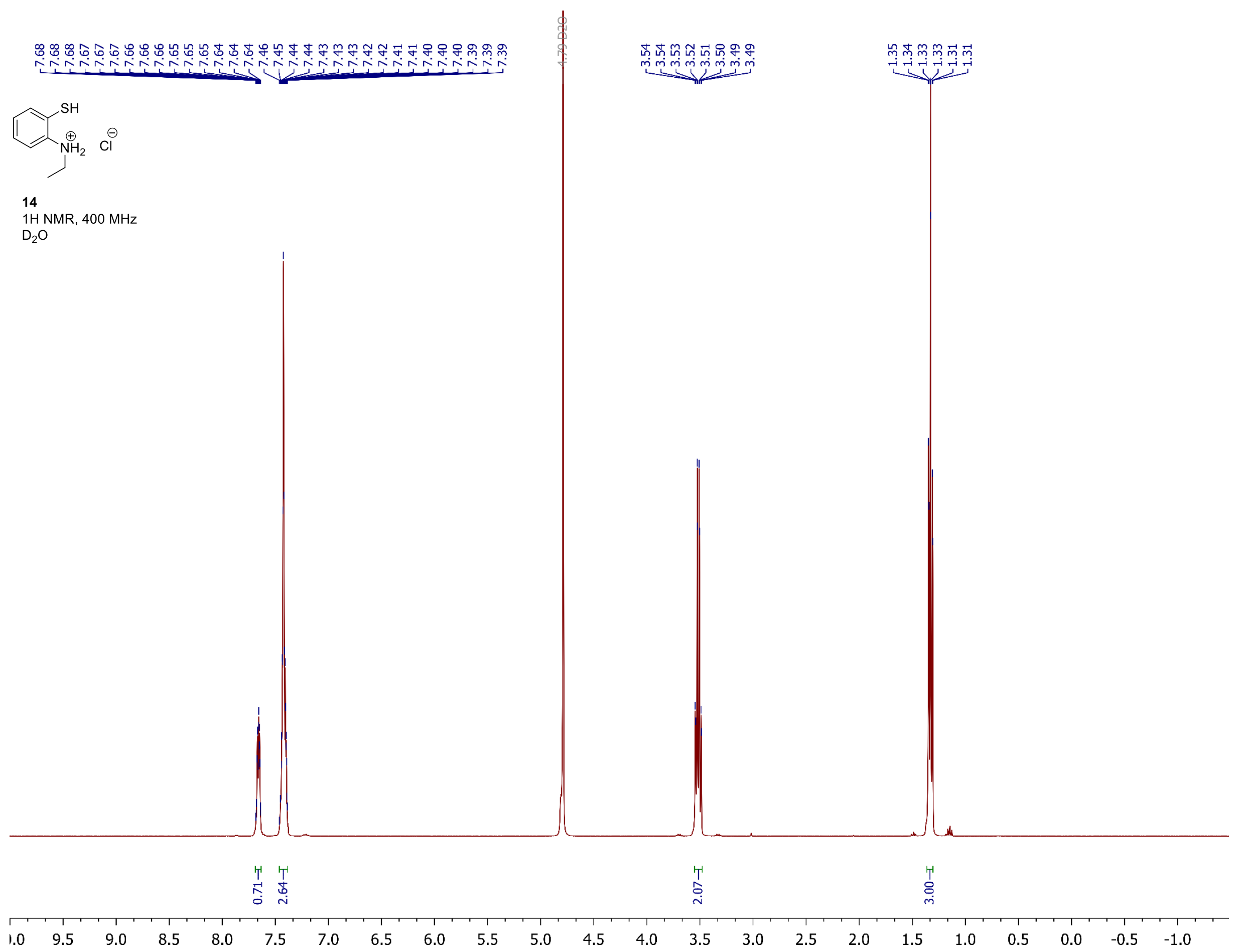




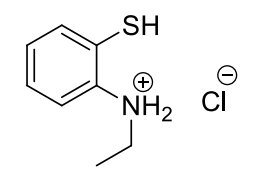

14

$13 \mathrm{C} N M R, 100 \mathrm{MHz}$

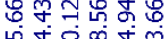

m

$\mathrm{D}_{2} \mathrm{O}$

i̊

咅

${ }^{13} \mathrm{C} \mathrm{NMR}\left(100 \mathrm{MHz}, \mathrm{d}_{2} \mathrm{o}\right) \delta 135.66,134.43,130.12,128.56,124.94,123.66,46.60,10.14$

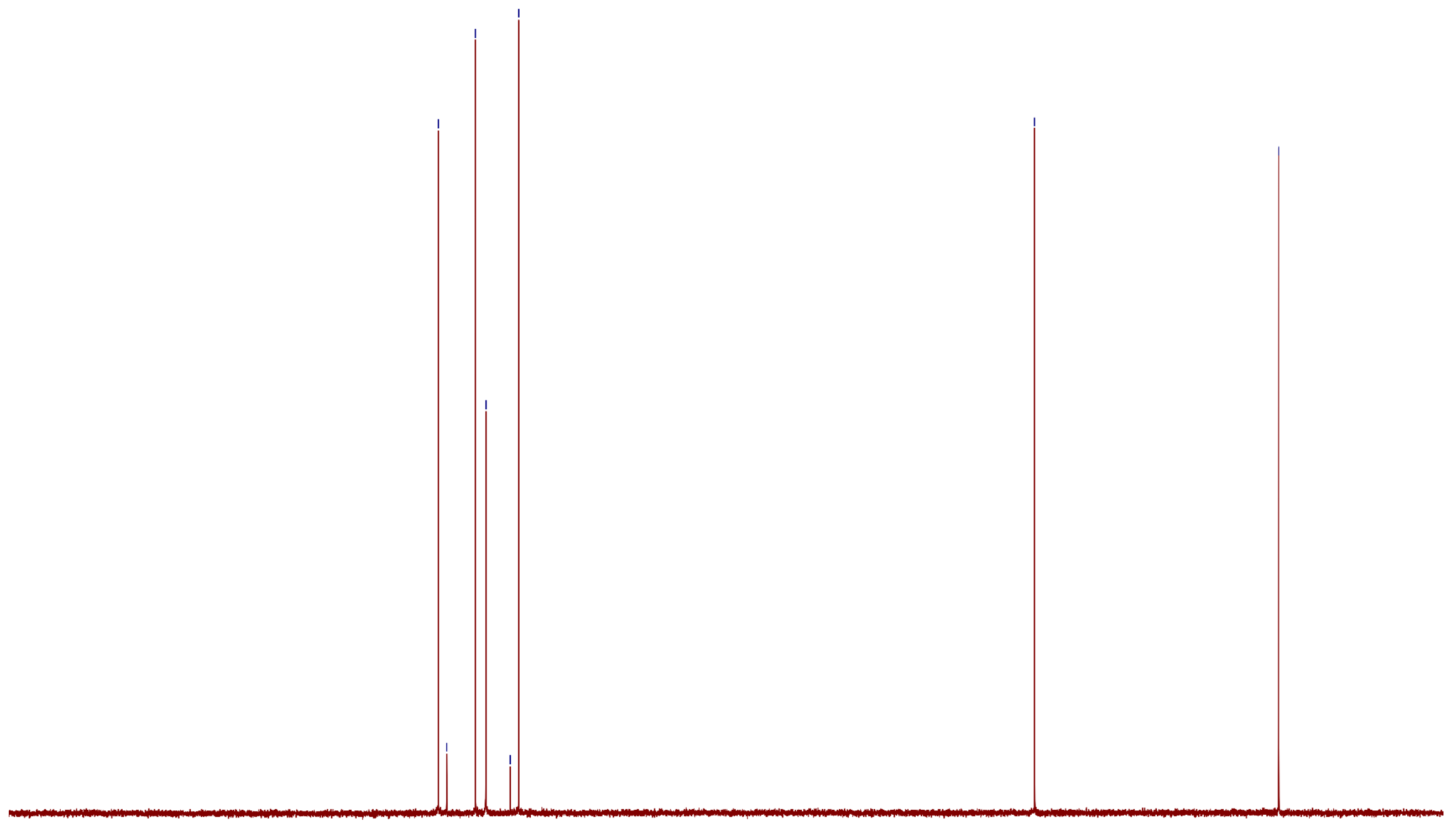




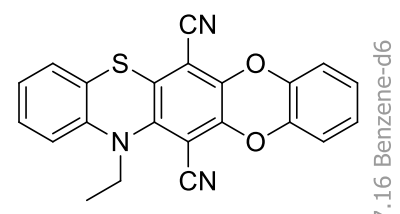

6

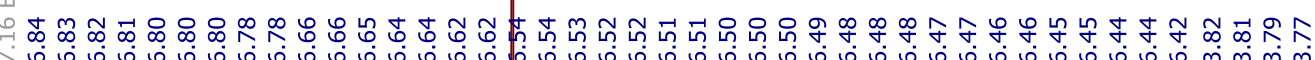

1H NMR, $400 \mathrm{MHz}$

$\mathrm{C}_{6} \mathrm{D}_{6}$

ம

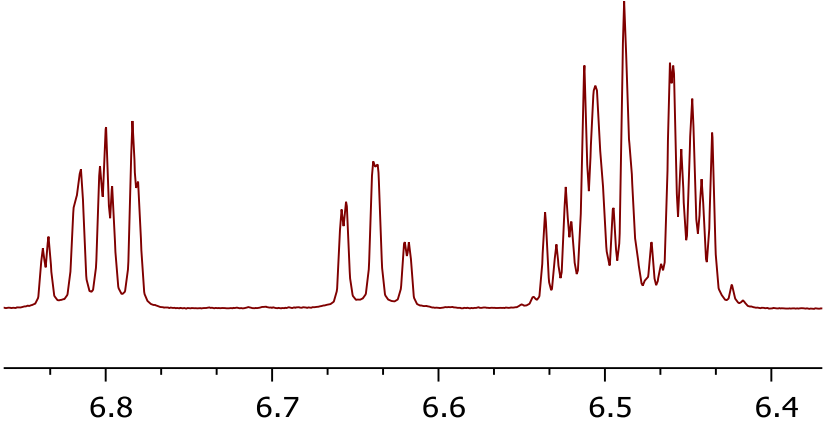

6.4

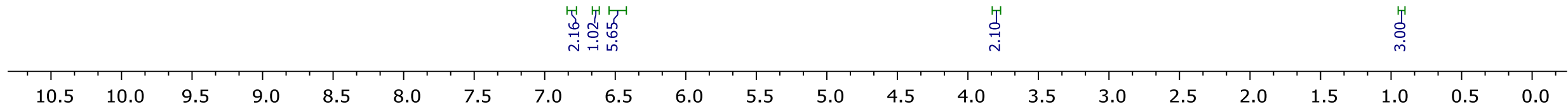




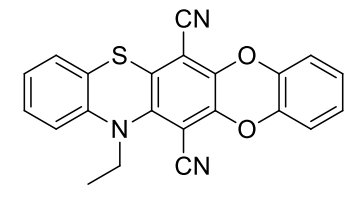

6

13C NMR, $100 \mathrm{MHz}$

$\mathrm{C}_{6} \mathrm{D}_{6}$

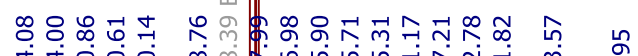

竎㠻守高宗

।

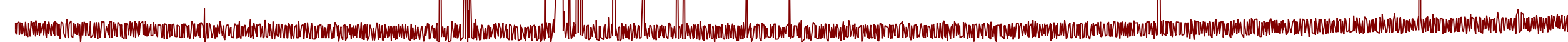

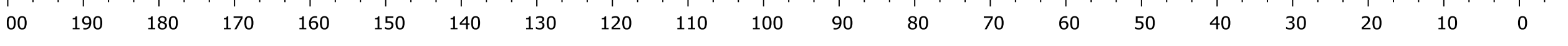



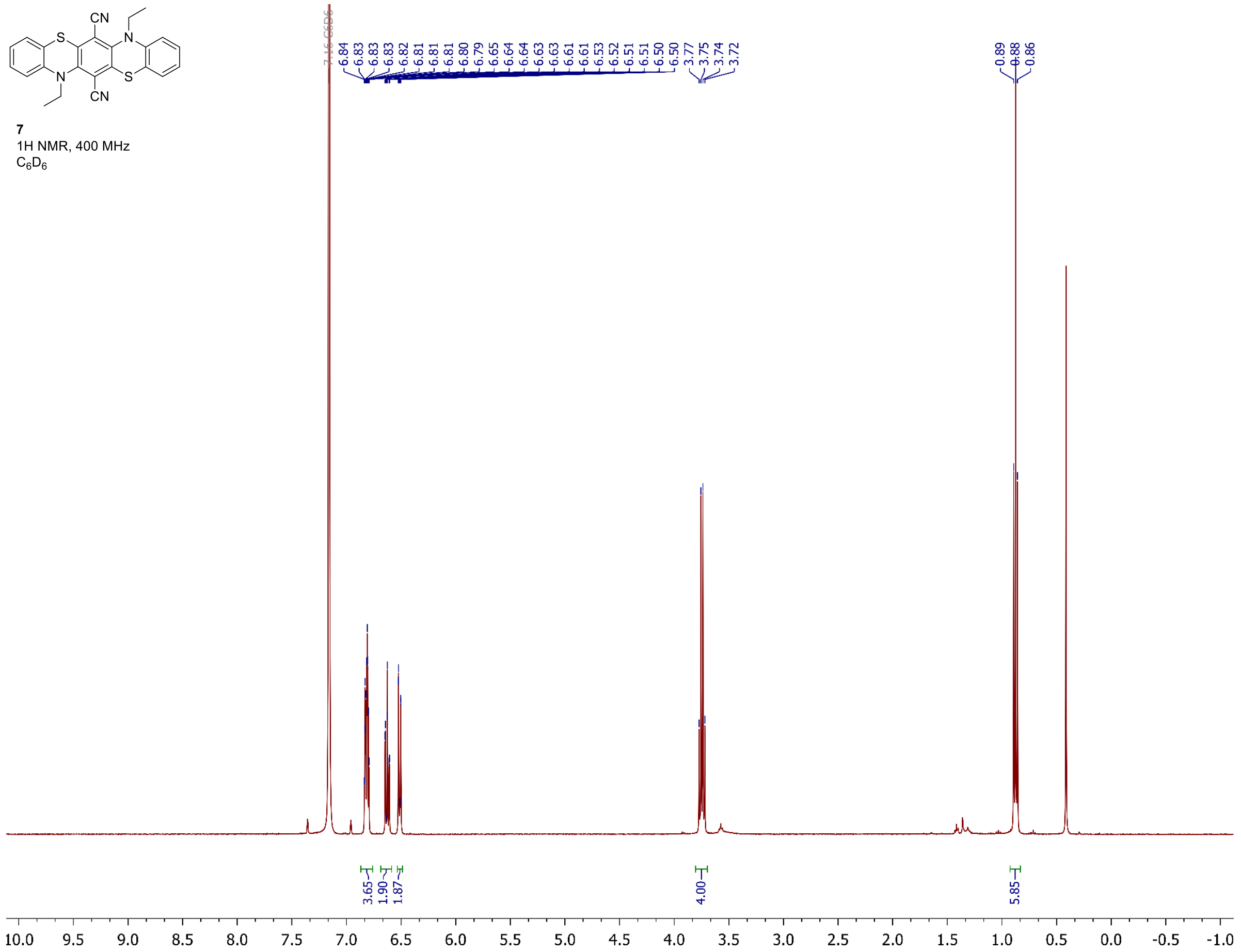


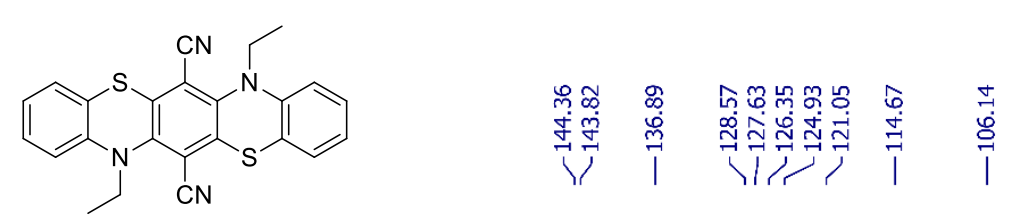

7

$13 \mathrm{C} \mathrm{NMR,}, 100 \mathrm{MHz}$

$\mathrm{CDCl}_{3}$

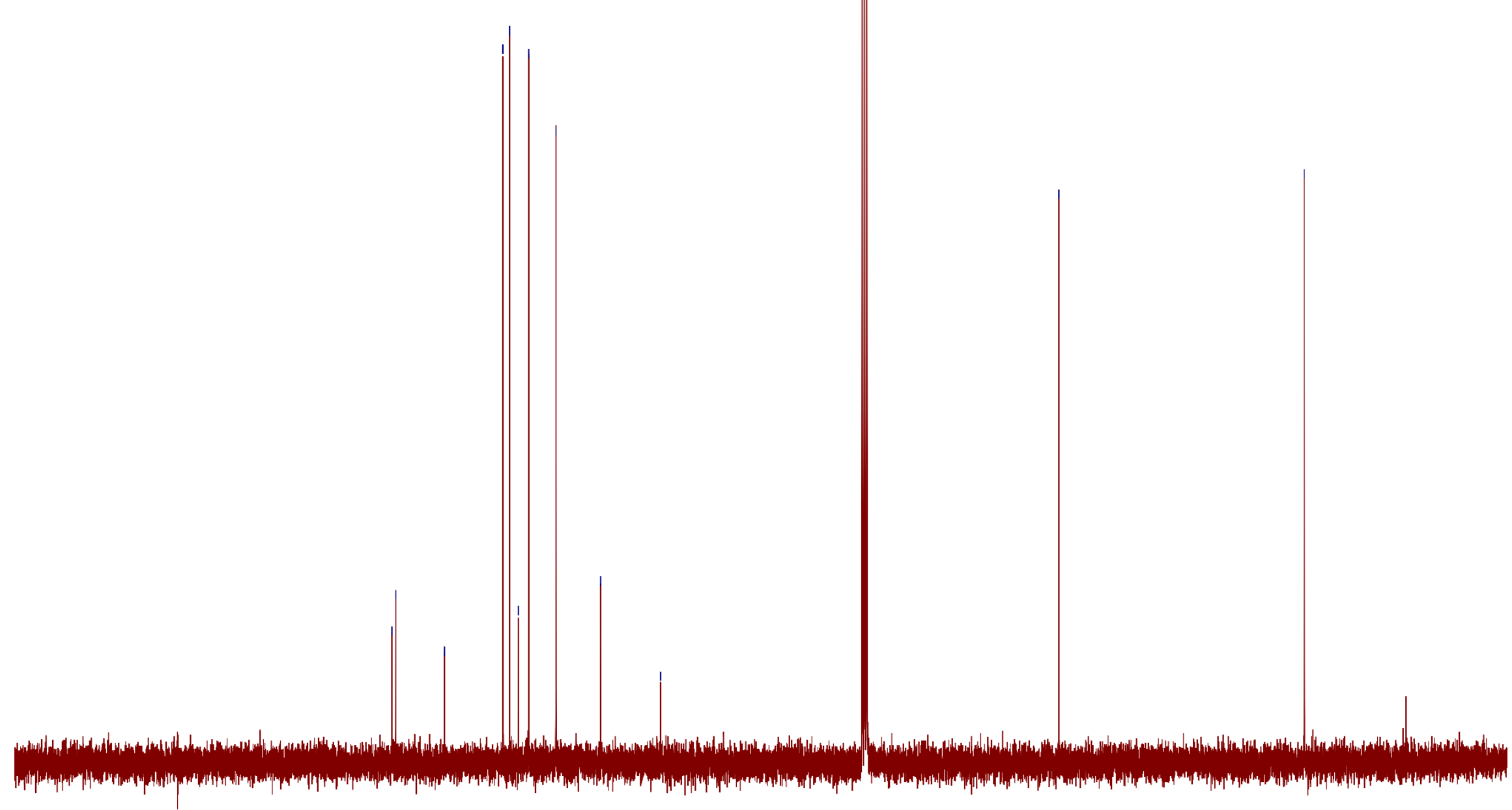

$\begin{array}{lllllllllllllllllllll}190 & 180 & 170 & 160 & 150 & 140 & 130 & 120 & 110 & 100 & 90 & 80 & 70 & 60 & 50 & 40 & 30 & 20 & 10 & 0 & -10\end{array}$

